\title{
Hash Based Co-Operative Method To Handle Sybil Attack Sequences In Vehicular Ad Hoc Networks
}

\author{
B. V. S. P. Pavan Kumar, S. S. V. N. Sharma
}

\begin{abstract}
Vehicular without any preparation arrange (VANETs) being stretched out to depict kept up for traffic control systems, stay away from misfortune oriented_information relations, stopping and information correspondence in remote structure structures. Protection and security are generally two _concerns in vehicular_ad hoc systems. Dreadfully,_VANET have absolute best privacy safeguarding ways to deal with oversee fragile Sybil assaults, where a malignant client can be familiar and indicate with be different vehicles. VANETs by making hallucination organized traffic deter. So that in this paper, we propose and develop Hash based Co-operative and Cryptographic Approach to handle Sybil attacks. This approach consists statistical approach to identify and verify vehicle information (id, ip address and where it is from and other details) and develop HMAC (Hash based Message Authentication Codes) encryption approach to detect Sybil attacks from forgery oriented attack sequences. Finally based on US map data available in VANET basic security web site, we generate different simulations to evaluate efficiency and feasibility of proposed approach. Our schema supports Sybil attacks detection without any support of outside positioning environment. Our experimental results show efficient result communication over detection of Sybil attacks in wireless network communication.
\end{abstract}

Keywords: Vehicular ad hoc networks, Position verification, Cryptography, Hashing and Sybil attack

\section{INTRODUCTION}

Vehicular Ad-Hoc Network (VANET) is a specific kind of Mobile Ad-Hoc Network (MANET) that gives correspondence between (1) neighboring vehicles and (2) vehicles and near roadside sorts of apparatus. VANETs are one way to deal with oversee execute Intelligent Transportation Network (ITS), a system for yielding information and correspondence movement to convey structure in addition, vehicles. It relies on IEEE 802.11p standard for Wireless Access for Vehicular Condition (WAVE). These systems have no settled structure, and they rely on themselves for seeing any structure handiness. A VANET is a decentralized structure as each middle plays out the sections of both host and switch. The standard incredible position of VANET correspondence is improvement of voyager thriving by exchanging forewarned messages between vehicles. VANETs move from MANETs

Revised Manuscript Received on August 14, 2019.

B.V.S.P.Pavan Kumar, Professor Malla Reddy Engineering College For Women Hyderabad, Telangana, India.(email ID: bvsppkumar@gmail.com)

Dr. S.S.V.N.Sharma, Professor, Vagadevi Engineering College, Warangal.Telangana, India.(email ID: ssvn.sarma1@gmail.com) in high convenientce of focus focuses, massive size of systems, geographically obliged topology, and tireless structure break. A monstrous bit of the assessment on VANET is based on Medium Access Control (MAC) layer and the system layer. VANETS intend to make applications, for instance, crash keeping away from, course evolving, and so on. _Security of vehicular systems is still to _a remarkable degree an inspected zone.

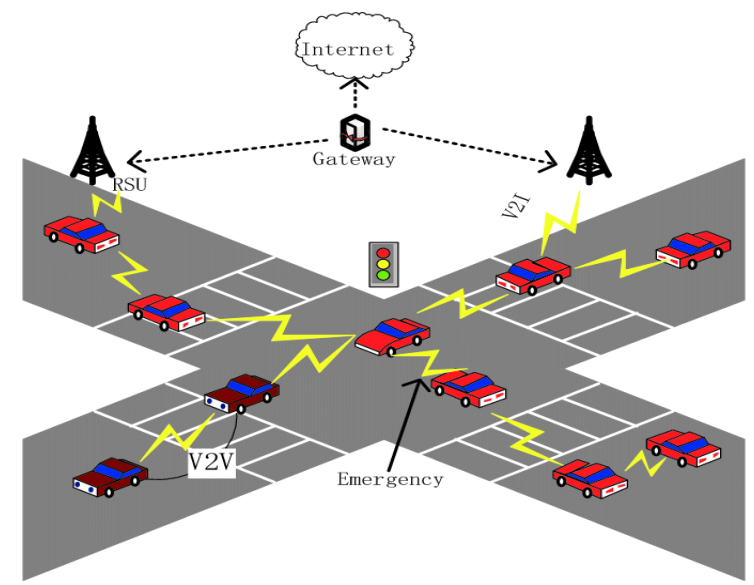

Figure 1. Generalized architecture of VANET.

VANET, being a remote compose, obtains all the security perils that a remote structure needs to control. VANET security is fundamental in light of the way in which that an insufficiently spread out VANET is weak against arrange ambushes, and this can deal the flourishing of drivers. A security system should ensure that transmission begins from a trusted in source and is genuinely not a changed in movement by various sources. It should in like manner hit an offset with affirmation in light of the way that executing security and assurance together in a structure is refuting. There are various types of potential strikes on VANETs. It is urgent that VANET security should be fit for managing every sort of ambushes. VANET security isn't proportionate to that of remote and wired systems because of its unfathomable characteristics of compactness objectives, establishment less structure, and brief term of connection between focus focuses. In a wired system, structure has isolates for express cutoff focuses, for example, switches pick the course to objective while structure has send and get messages. Security use is tolerably fundamental as systems

Published By:
Blue Eyes Intelligence Engineering \& Sciences Publication 


\section{HASH BASED CO-OPERATIVE METHOD TO HANDLE SYBIL ATTACK SEQUENCES IN VEHICULAR AD HOC NETWORKS}

ought to be physically adjusted for listening stealthily. Remote structures use infrared or radio intermittent signs to offer among devices. These systems can be either (a) structure based or (b) establishment less. Structure make remote systems are based concerning Public Switched Telephone Network (PSTN) switches, MSCs, base stations, and versatile hosts. In extraordinarily designated systems, a sort of structure less remote systems, focus focuses play out all exercises, for instance, arranging, pack sending, and system affiliation, and so on. The present security plans use standard motorized etching and declarations using Public Key Infrastructure (PKI). In VANETs, key reason for get together of security is on progress related applications. Non security applications have less stringent security necessities. There is no prior trust connection between the focuses of VANETs in light of its establishment less nature. Any middle can join and leave the system at whatever point without indicating various focuses in locale. Enchanting security plans are legitimately productive in a situation making can be seen through joint exertion between the no of focuses by persevering through that greater piece of focuses are quick. So that in this paper, we propose and make Hash based Co-usable and Cryptographic Approach to oversee Sybil ambushes. This framework includes quantifiable way to deal with oversee perceive and attest vehicle data (id, ip address and where it is from and different subtleties) and make HMAC (Hash based Message Authentication Codes) encryption way to deal with oversee see Sybil assaults from mutilation engineered strike movements. This structure perceive that vehicles talk with one another in a multiricochet way, and the correspondence is seen by a RoadSide Box (RSB) through idle getting. The RSB is securely connected with the Department of Motor Vehicle (DMV) by methodologies for a backhaul wired structure. The DMV fills the activity of an affirmation ace (CA), and can arrange vehicle enlistment, ownership, and other definitive structures. Our course of action requires the DMV to furnish vehicles with a pool of aliases are used for concealing the vehicle's brilliant character. On the other hand, to shield a vehicle from using various nom de plumes a Sybil strikes, the nom de plumes to a particular vehicle are hashed to a conventional regard. By figuring the hashed estimations of the got assumed names, RSB and the DMV will probably pick if the normal names from a similar pool, along these lines distinguishing a Sybil attack. Though our calculation requires the count/stockpiling of the pen names, demonstrate that the computational overhead and capacity overhead are moderate.

\section{RELATED WORK}

Sourav Kumar Bhoi et.al Vehicular correspondence is a considerable region of research in the field of vehicular innovation. The improvement of programming and equipment in correspondence frameworks execute to the engendering of new networks. The principle thought behind utilizing this new innovation is to make a mishap free condition. New plans, conventions and usage are utilized as a part of vehicular specially appointed network (VANET) to give Intelligent Transportation Services. It is likewise rethought the motivation behind VANET which gives administrations to the clients. The principle rationale of this investigation territory is to examine the prevalent thoughts in vehicular correspondence. YUN-WEI LIN et.al Vehicular Ad hoc Network (VANET), a blueprint of adaptable spontaneous coordinates (MANETs), is an ensuring segment for the able transportation structure (ITS). The course of action of sorting out shows up in VANETs is central and essential issue for the heavenly ITS.

Mina Rahbari et.al Vehicular trades recognize a fundamental part in giving security transportation by techniques for prospering message transmission. Researchers have pondered various responses for verifying security messages. Shows in light of a settled key establishment are dynamically versatile in execution what's more oversee more grounded security. The request of this paper address a methodology in light of a settled key establishment for presentation mimic ambushes, so to represent case Sybil assaults, in the vehicular phenomenally appointed structure. This ambushes puts a great effect on the execution of structure. The proposed method, using a cryptography instrument to see the Sybil ambushes. Finally, using Mat lab test engineer the consequences of this methodology are reconsidered, This structure has low delay for perceiving confirmation Sybil ambushes, in light of how most assignments are done under Certification Authority, so this model is a gainful technique for assertion Sybil strikes.

Manjunatha T. N et.al Due to pass on Wireless Sensor Networks (WSNs) and reducing of progress safe apparatus, security in sensor sort out is the credible impediment. Research is being done on a few security attackss on remote sensor structures. Remote Sensor Networks are vivaciously grabbing interests of authorities from the academic world, industry, making movement and gatekeeper. WSNs consolidates enormous number of sensor focus focuses and a minor sink focus focuses or base station are extended in the field to amass information about the class of physical world and transmit it to hypnotized customers, routinely which are used as a touch of usages, for instance, living space watching, military reconnaissance, condition seeing and flourishing checking. Right when an inside point misguidedly conveyed various characters or claims fake id, is called Sybil assaults. In Any system it is particularly delicate to the Sybil ambushes where in a deadly focus issue the best development of the structure. This paper target considered blend of security issues, security threats, Sybil ambushes and various techniques to expel Sybil assaults.

Smash Shringar Raw et.al Vehicular Ad hoc Networks (VANETs) is the testing way to deal with give security to different applications and the drivers and additionally travelers. It turns into a key part of the wise transport framework. Considerable measures of works have been done towards it however security in VANET got less core interest. In this, we have talked about the VANET and its specialized and security viewpoints. We have additionally talked about some significant attacks and arrangements that can be executed against these attacks. We have analyzed the arrangement utilizing different parameters. Finally we have depicted the components that are utilized as a part of the

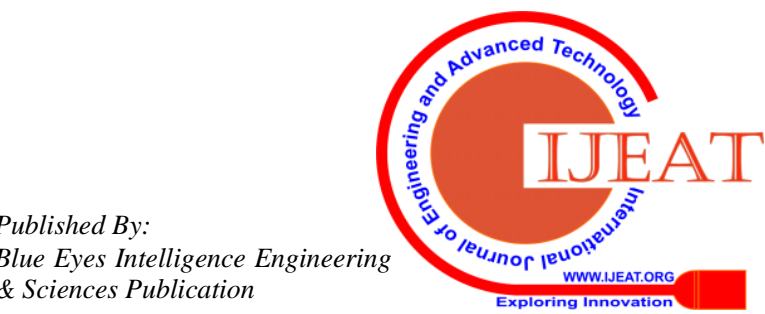


arrangements.equation. Use "(1)", not "Eq. (1)" or "equation (1)", except at the beginning of a sentence: "Equation (1) is ..."

\section{SYBIL ATTACKS IN VANETS}

Vehicular specially appointed networks are a promising new innovation that can give monetarily down to earth arrangements and advantages to an assortment of uses for the vehicle framework, for instance position detecting, movement observing, clever transport framework and so forth. Vehicular Ad hoc Networks are considered to can possibly not just help in the basic leadership for the drivers, for example, course determination to the goal that is the best, less congested course, yet in addition to enhance and travelers very much educated about the road and improvement conditions and any fiascoes ahead. Thinking about the criticalness of vehicular astoundingly relegated systems masters have constantly been in mission to raise any security risks that VANETs are confronting, which may diminish the capability of vehicular structures and even make hurt life thriving. Security is required for unending especially relegated system applications, especially when the vehicular unrehearsed structures are in charge of guaranteeing information or checking movement or transmitting vital lifesaving information. Security in vehicular exceptionally alloted systems is baffled by the offered thought of the remote correspondence and a bit of the portions related to the middle focuses' conveyability lead. Alongside these complexities, vehicular focuses have extensive cutoff and computational resources which could be noteworthy to the degree making security models. Vehicular ad libbed creates (VANETs) address complex appropriated systems especially like that of adaptable astoundingly named structures including remote supportive focuses. Procedure for Sybil assaults in remote systems appear in figure 2 . These inside focuses can self-structure themselves into various random driving force topologies always and uninhibitedly. This associates with and wants the vehicular focus focuses to speak with one another in spots where there is no correspondence structure presented. All in all, correspondences in remote systems are done in light of a novel identifier that should addresses a structure substance which we propose as a middle point. In a system these identifiers are used for looking out for the structure substance in the midst of correspondence, thusly enveloping a sensible mapping between a section and a character.

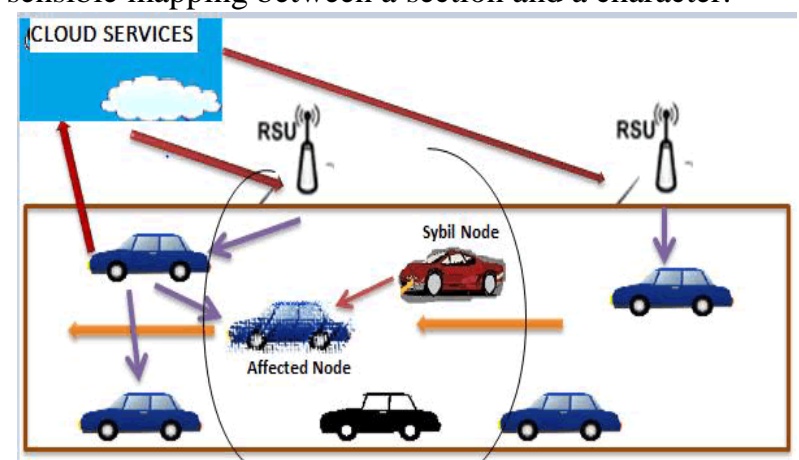

Figure 2. Resistant Sybil attacks cryptography specification in VANETs.

This is a general doubt diverse show sections expect either unquestionable or unequivocally, establishing that two characters address two clear focus focuses. Regardless, the nonattendance of such keeping an eye out for systems in vehicular extraordinarily picked structures makes the soft spot for Sybil snare, which concludes that a risky vehicle can ensure specific fake characters wound up being hazardous to different vehicular structure applications. In deals to appreciate the strike and its enormity in vehicular structures let us consider one of only a bundle couple of conditions. A driver inspecting for more resources or action free road to his objective can devise that a broad number of vehicles are voyaging circumscribing, thusly making a dream of headway stop up. Various vehicles tricked by this fantasy pick an emotionally supportive network and draw eventually from the road, therefore giving the attacker a stop up free imaginative (to the degree sort out information move limit, etc.) course. All things considered, all these made vehicles remain influenced by attacker, so a couple of various applications or structure shows may in like manner be influenced by such strikes depending upon aggressors' yearning. A part of this breaker deviation from truth by influencing the results of law based shows using Sybil focus focuses. Sybil focus focuses can other than be used to dispatch Denial of Service strikes that can hurt the assignments of system, leaving other genuine focus focuses be for favored position by influencing data spread shows. Correspondingly attacker can in like manner use Sybil focus focuses for blocking life essential information causing real thriving threats. For example, if a vehicle is using an application for early reproving, and another vehicle two focus focuses ahead in a similar area diminishes its speed absolutely or related breaks, a pass on message will be made offering forewarned to the going with vehicles. This message at whatever point got by some Sybil focus point can destroy the sending strategy likewise leaving the going with vehicles at an astounding peril or hazard. This could achieve enormous occurrence on the roadway, potentially causing amazing loss of life. Sybil ambushes address an outstanding danger without joined character relationship in vehicular phenomenally named structures. For the security show to energize there is a requirement for a noteworthy, undeniable, and relentless character per focus point.

\section{PROPOSED APPROACH}

Usually Sybil attacks have three different categories, namely resource testing, registration for identification and verify based on position. Resource testing requires multi channel data access, registration along with node/ vehicles identification and verification need to detect each node position based on ip address and port based parameters present in network configuration. Module implementation for proposed approach is as follows:

Hash based Co-usable Method: This system sees potential Sybil spotlight centers subordinate around position with id, name and other parameter check, center point signal quality with different properties. we depict three classes of focus focuses' parts: claimer, witness, and verifier. Each inside point would only from time to time imagine these parts, that

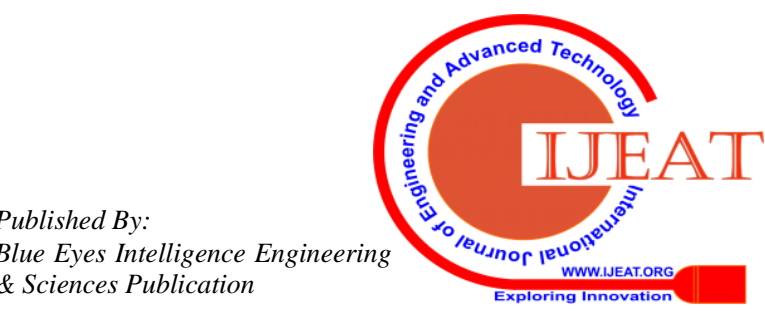




\section{HASH BASED CO-OPERATIVE METHOD TO HANDLE SYBIL ATTACK SEQUENCES IN VEHICULAR AD HOC NETWORKS}

is, each middle point is a claimer, a passerby moreover a verifier yet at different minutes and for different purposes.

1.Claimer. Each inside point from time to time yields a guide message at reference point between times, tb, with the veritable point of convergence of neighbor divulgence. In the reference point message, it ensures its character and position, for instance, GPS position. At the present time, we name inside point is named as a claimer. The target of our system is to check its clarified position.

2.Witness. Each and every neighboring center point, inside the standard level of the claimer, would get the past sign message. They measure the pennant quality and extra the relating neighbor information in their memory. Next time they give a reference point message, they will join their neighbor list, including the pennant quality estimations for each got reference point, to the guide message. We name these inside centers performing estimations and showing estimations as eyewitnesses.

3.Verifier. We call an inside point performing position attestation is a verifier. Happening as expected to getting a reference point message, an inside point holds tight for a checking between time, TV, in the midst of which it gathers enough banner quality estimations concerning the past reference point message from neighboring onlookers. TV may be to some degree longer than the reference point break $\mathrm{tb}$, since after another between time of tb, each neighboring onlooker should have passed on a sign containing the standard estimations. With the amassed estimations, the center (verifier) can locally pick a run of the mill position of the claimer. By then, inside point separates the laid out position and the beginning late ensured position of the claimer. If the fragment defeats a predefined limit ?, the claimer is seen as a Sybil center point.

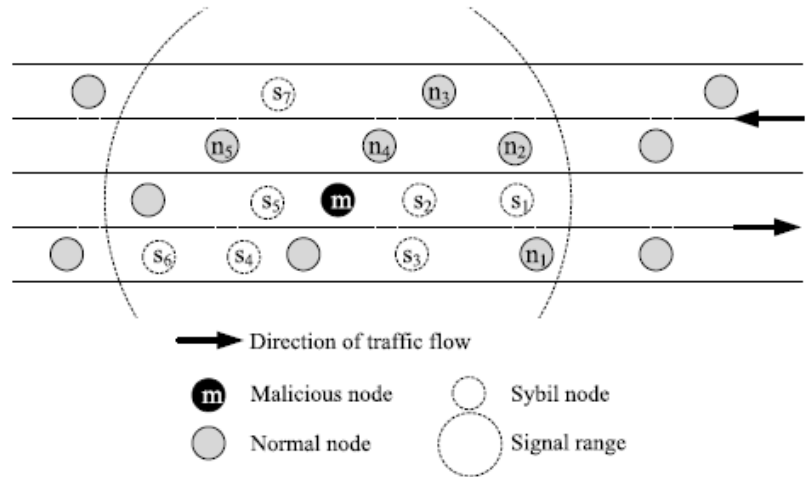

Figure 3. Proposed approach architecture with different modules.

We take Fig. 3 for example. Center point s1, a claimer_(a Sybil center point), presents a_ signal, ensuring its character and position. Center point $\mathrm{n} 1$, _a verifier, assembles _all flag quality estimations from neighboring onlookers which have gotten the guide. Plainly, the last studied position of $\mathrm{s} 1$ would be near the state of center $m$, as opposed to the position s1 ensured, as center s1 and female horse physically a similar vehicle.

The message can be position in following manner

\{NodeID, Beacon\#, Position, NebList, Signature\}

NebList : $\{$ NodeIDi, Beacon\#i, RSSi\},

Where NodeID is the claimer's character, Beacon\# is a guide development number, Position is the sender-declared position, NebList is the sender's most recent neighbor onceover containing signal quality estimations, and Signature is the pushed etching for the whole assembling. In everything of NebList, RSSi is the Received Signal Strength of reference point Beacon as of late got from neighboring hub NodeIDi. Next section, we discuss about estimated position detection at different positions at different simulation parameter sequences. For that, to improve robustness of detection of Sybil attacks in VANETs.

\section{Random Consensus Calculation:}

We propose this calculation to increase or improve estimation of positions of different vehicles. This method mainly used to design computer graphics and artificial intelligence oriented applications to improve robustness of network systems. Using this approach, we adopt Sybil attacks detection in VANETs.

Input: $\mathrm{n}$ flag quality estimations and
comparing positions
Output: Estimated position of the subject
$1 \mathrm{~S} \leftarrow \emptyset ; /$ Set of accord sets
2 while $\mathrm{I} \leq \mathrm{f}$ do
$3 / / \mathrm{fis}$ number_of trials
$4 \mathrm{~s} \leftarrow$ random(b);//instate an arbitrary
agreement set of estimate b
5 while error(s) $\leq \gamma$ do
$6 \mathrm{r} \leftarrow$ arg $\operatorname{minu} \in \mathrm{s}\{$ error(s $+\{\mathrm{r}\})\} ;$
$7 \mathrm{~s} \leftarrow \mathrm{s}+\{\mathrm{r}\} ;$
8 end
$9 \mathrm{~s} \leftarrow \mathrm{s}-\{\mathrm{r}\} ;$
$10 \mathrm{~S} \leftarrow \mathrm{S}+\{\mathrm{s}\} ;$
11 end
$12 \mathrm{~s} \leftarrow$ arg maxs $\in \mathrm{S}\{\operatorname{size}(\mathrm{s})\} ;$
13 retum get_model_position(s);

Algorithm 1. Ransom consensus calculation procedure to process position of vehicles.

The proposed estimation is shown in Algorithm 1. The figuring has all the standard quality estimations and tolerating a gander at positions as information and has the overviewed position of the subject as yield. An understanding set contains undeniable flag quality readings. Any understanding set strikingly fits a flag quality stream show up inside a given goof extend, ?. S is a course of action of contender accord sets, which is instated to be ? at stage 1 . Stages 2-10 join a circle, which is performed $\mathrm{f}$ times (number of fundamentals). The circle is required to discover f contender getting sets, all of which watches out for a model. Near the start of the buoy (at stage 3), a one of a kind set, s, is instated with as less information as could be customary in light of the present circumstance, where, $b$ ? $n$, and $\mathrm{n}$ is certainly the amount of standard quality readings. The set, s, is reached out at stages 4-7 with the need that the fumble, how s fits a model, isn't commonly a predefined destruction up, ?. Accordingly, in each circle, we begin from _a irrelevant sporadic set_and find _the most basic understanding set inside bungle ? . _At long last, at stage 11 ,we can find the best understanding set in $\mathrm{S}$. In context on 
this methodology, learn mean square misunderstanding utilizing reviewed position of vehicle _or focus point chose as looks for after:

$$
\operatorname{MSE}(s)=\frac{\sum_{i=1}^{n}\left(s\left(w_{i}\right)-R S U_{p}\left(w_{i}\right)\right)^{2}}{n}
$$

Where _ $\mathrm{p}$ is an arranged spot of the claimer, $\mathrm{k}$ is _the variety of spectators, $s$ is _the set of sign robustness numbers (s(wi) shows the sign_durability learning at watch wi), Road Side Unit (RSUp) (wi) is the sign quality at wi expelled from the sign quality spread model. _By differing $\mathrm{p}$, we can decrease MSE and in end get the perfect surveyed place ^p. After measure position of vehicle then _encrypt vehicle nuances for acknowledgment of Sybil attacks in VANETs.

\section{Hash Based Prevention:}

In this stage, when vehicles communicate, the RSUs catch every one of the vehicles that are inside their correspondence run. We expect that the RSUs posses the keys from the Data Map Vector (DMV), and can consequently process grained hash estimations of a given nom de plume. At the point when all occasions with time have been gathered, the RSU experiences every nom de plume registers the grained hash esteem. In the event that hash estimations of pen name equivalent, at that point the RSU sees that there are no less than two nom de plumes the same grained hash esteem used to sign the occasion. This can be a Sybil attack where one vehicle is utilizing different pen names report a similar occasion.

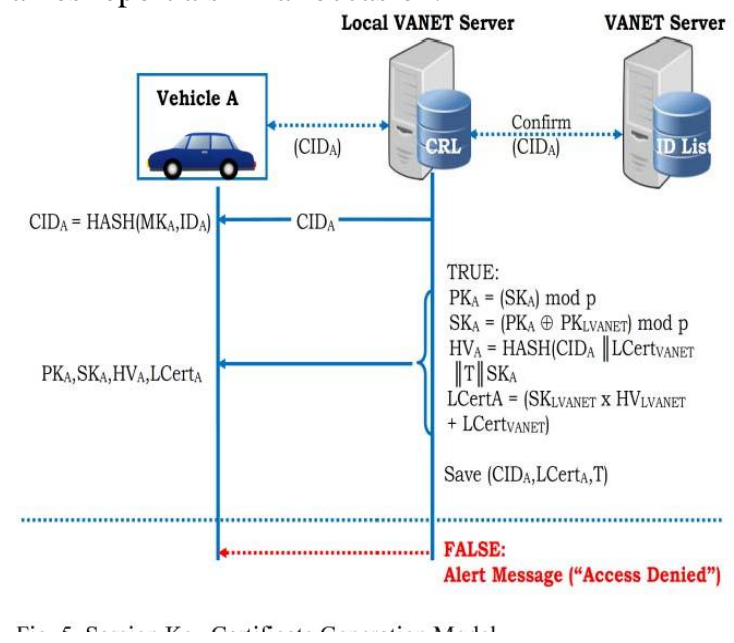

Figure 4. Detection procedure for proposed approach.

In the subsequent stage, on getting a RSU report, the DMV first affirms the imprints to keep an exchanged off RSU from catching a liberal vehicle appeared in above figure 4. In case the RSU ends up being fulfilled, the DMV stores the fine-grained hash an impetus for each nom de plume in the RSU report. If past hash regard and current hash regard is same then the DMV presumes that the two nom de plumes from a comparative vehicle that has tried a Sybil assault. The DMV, find the dangerous vehicle from the handled puzzle plate number, and takes also exercises.

\section{PERFORMANCE EVALUATION}

Experimental Setup: Experimental simulations were carried out in JAVA using MAC 802.11/16 networking. In order to decrease packet overhead, use secure hash calculation cryptography to store vehicle information in signature format. Basic summery of simulation parameters shown in Table 1.

\begin{tabular}{|c|c|}
\hline Parameter & Value \\
\hline Distance of Simulation & $500-100 \mathrm{~m}$ \\
\hline No. of vehicles & $\begin{array}{c}\text { Based on random } \\
\text { consensus iterations }\end{array}$ \\
\hline Speed & $50-500 \mathrm{~km} / \mathrm{h}$ \\
\hline Encryption & SHA 192 bits \\
\hline Bandwidth & $2 \mathrm{MB}$ \\
\hline
\end{tabular}

Table 1. Simulation parameters.

Using above simulation parameters, Design simulation window shown in figure 5.

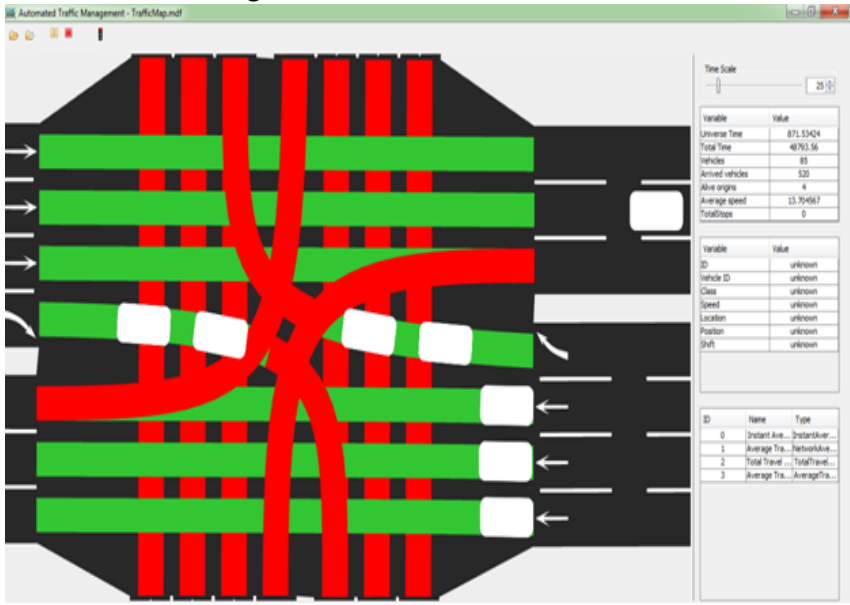

Figure 5. Simulation window set for vehicle data transmission.

The above figure shows basic design of simulation set up to form communication between RSU server and different vehicles at different positions shown in right side of the above figure five. Hash based DMV storage data shown in figure 6.

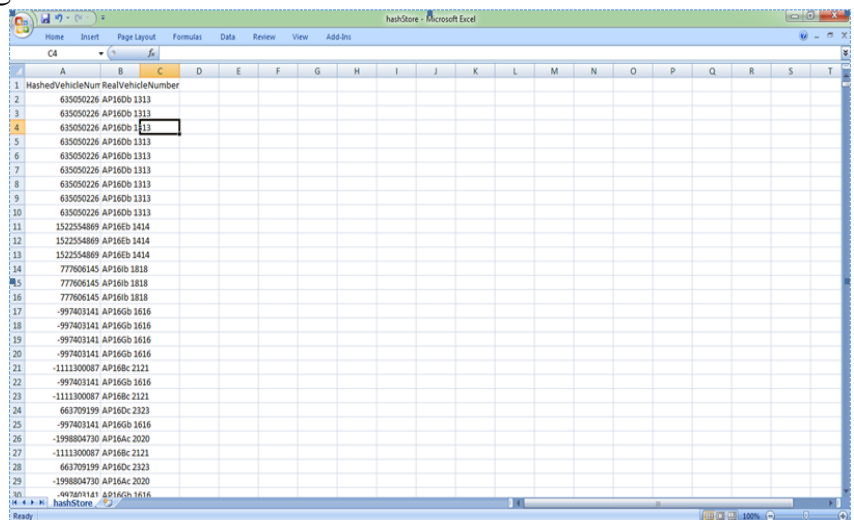

Figure 6. Secure DMV data storage to detection of Sybil attackers in VANETs.

Figure 6 shows secure data of Sybil attacker's vehicles on server side to identify which vehicle perform attack on overall VANET with communication between different vehicles in wireless communication.

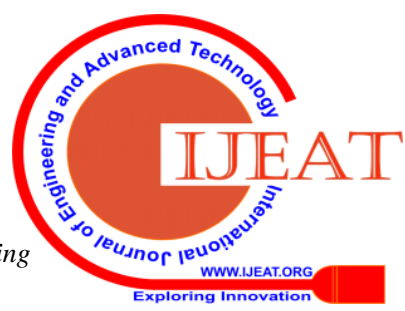



HOC NETWORKS

\section{RESULTS}

Based on discussion present in section 5.1, we define different parameters like detection accuracy and time in data communication between different vehicles in VANETs. Figure 7 show the detection accuracy of proposed approach with respect vehicles in sequential data communication at different vehicle positions at different window co-ordinate values.

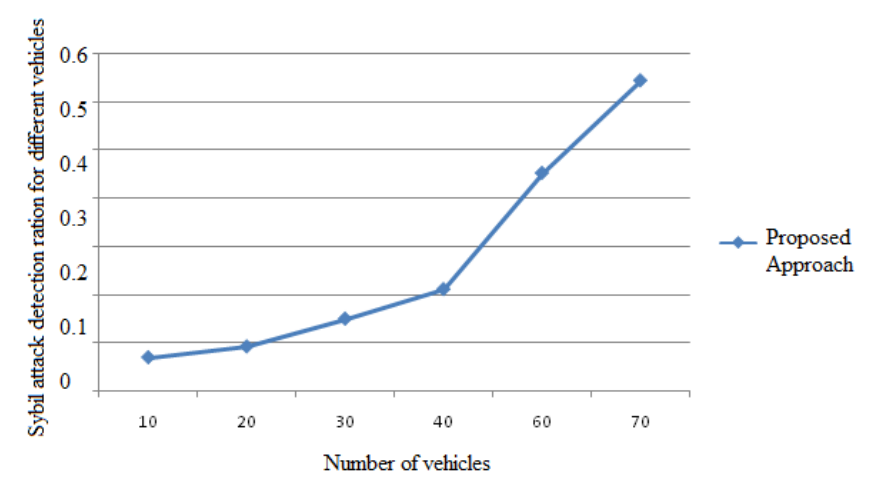

Figure 7. Sybil attack detection accuracy for different vehicles.

Sybil attack detection time analysis shown in figure 8 with different vehicles with different positions.

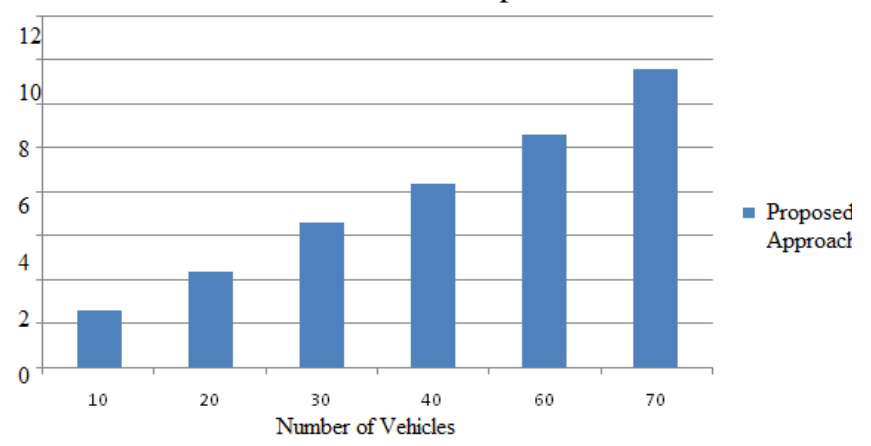

Figure 8. Sybil attack detection time efficiency for different vehicles.

By using hash key allocated program and light-weight and scalable structure perform, we are helping the efficiency of VANETs and discovering the Sybil strikes in VANETs. We take reviews of automobiles details from DMV and held in RSU. Consider two RSUs automobile details, if any automobile efforts in RSU using hash key submission then that automobile as consider as enemy. And also we are helping the efficiency of VANET from Sybil attacks.

\section{CONCLUSION}

In this paper, we propose a Hash based Co-usable and Cryptographic Approach to handle Sybil attacks. In proposed framework, the Department of Motor Vehicle furnishes vehicles with an exceptional pool of aliases, for concealing a vehicle's one of a kind character. Two parameter definitions: direction time confine: time to navigate between 2 RSUs direction length restrain: the \# of RSU crossed in a period. Propose a security utilizing SHA where vehicles recognize Sybil attacks agreeably in protection saved VANETs. To keep a vehicle from mishandling the nom de plumes dispatch a Sybil assault, hashing is utilized. By utilizing hashing, vehicle nom de plume hashed and put away in street side units (RSU). Presently RSU ascertain the hashed estimations of caught pen names, decide whether the pen names from a similar pool - provided that this is true, it presumes a Sybil assault. An entire security convention which incorporates testing, affirmation and isolate are portrayed. Our proposed pattern fundamentally centers on recognizing Sybil assault and gives security. Furthermore we discuss about Denial of Service (DOS), (Distributed DOS) attack sequences in VANETs. It is our future work, use some framework oriented scenario to handle DOS \& DDOS attacks in VANETs

\section{REFERENCES}

1. Bo Yu, Cheng-Zhong Xua, Bin Xiao, "Recognizing Sybil ambushes in VANETs", J. Parallel Distrib. Comput. 73 (2013) 746-756.

2. Ali Akbar Pouyan, Mahdiyeh Alimohammadi," Sybil Attack Detection in Vehicular Networks", Computer Science and Information Technology 2(4): 197-202, 2014.

3. Tong Zhou, Romit Roy Choudhury, Peng Ning, and Krishnendu Chakrabarty," P2 DAP - Sybil Attacks Detection in Vehicular Ad Hoc Networks", IEEE JOURNAL ON SELECTED AREAS IN COMMUNICATIONS, VOL. 29, NO. 3, MARCH 2011.

4. Muhammad Saad Naveed* , M Hasan Islma, "Distinguishing proof of Sybil Attacks in Vehicular Ad Hoc Networks", Universal Journal of Communications and Network 3(1): 15-25, 2015.

5. Mina Rahbaril and Mohammad Ali Jabreil Jamali, "Successful DETECTION OF SYBIL ATTACK BASED ON CRYPTOGRAPHY IN VANET", International Journal of Network Security and Its Applications (IJNSA), Vol.3, No.6, November 2011.

6. H. Wang, J. Wan, R. Liu, "An epic expanding strategy subject to RSSI," Energy Procedia, Vol. 12, No. 1, 230235, 2011.

7. B. Yu, , C. Z. Xu, B. Xiao, "Recognizing Sybil ambushes in VANETs," Journal of Parallel and Distributed Computing, Vol. 73, No. 6, 746-756, 2013.

8. S. Abbas, , M. Merabti, , D. Llewellyn-Jones, K. Kifayat, "Lightweight Sybil Attack Detection in MANETs," IEEE, Systems Journal, Vol. 7, No. 2, 236-248, 2013.

9. C.- H. Ou, "A roadside unit based restriction contrive for vehicular uncommonly selected frameworks," Int. J of Communication Systems Wiley, No. 51, 123-130, 2012.

10. J. T. Isaac, , S. Zeadally, J. S. Camara, "Security attacks and responds in due order regarding vehicular uncommonly delegated frameworks" Communications IET, Vol. 4, No. 7, 894-903, 2010.

11. P. Y. Shen, "A viable open key organization framework for vehicular uniquely delegated frameworks (VANETS)," Masters by Research recommendation, Queensland University of Technology, 2011.

12. K. Ibrahim, "Data collection and dispersing in vehicular uncommonly delegated frameworks," Doctoral proposition, Old Dominion University, Norfolk, Virginia, 2011.

13. M. A. Razzaque, A. Salehi, S. M. Cheraghi, "Security and Privacy in Vehicular Ad-Hoc Networks: Survey and the Road Ahead," Springer Berlin Heidelberg, In Wireless Networks and Securit, 107-132, 2013. 
14. S. Chang, , Y. Qi, H. Zhu, J. Zhao, X. Shen, "Impression: recognizing Sybil attacks in urban vehicular frameworks," IEEE Transactions on Parallel and Distributed Systems, Vol. 23, No. 6, 1103-1114, 2012.

15. S. Park, B. Aslam, D. Turgut, C. C. Zou, "Prepare for Sybil attack in the fundamental course of action period of vehicular extemporaneous framework reliant on roadside unit support," Security and Communication Networks, Vol. 6, No. 4, 523-538, 2013.

16. T. Zhou, , R. R. Choudhury, P. Ning, K. Chakrabarty, "P2DAP-Sybil Attacks Detection in Vehicular Ad Hoc Networks," Selected Areas in Communications, IEEE Journal, Vol. 29, No. 3, 582-594, 2011.

17. M. Demirbas, Y. Tune, "A RSSI-based arrangement for Sybil ambush area in remote sensor frameworks," In Proc. Of International Symposium on a World of Wireless, Mobile and Multimedia Networks, 564-570, 2006.

18. S. Zhong, , L.E. Li, Y.G. Liu, Y.R. Yang, "Security holding zone based organizations for adaptable customers in remote frameworks," Technical Report. YALEU/DCS/TR-1297, Department of Computer Science, Yale University, 2004.

19. S. Abbas, , M. Merabti, , D. Llewellyn-Jones, K. Kifayat, "Lightweight Sybil Attack Detection in MANETs," IEEE, Systems Journal, Vol. 7, No. 2, 236-248, 2013.

20. B. Dutertre, , S. Cheung, J. Toll, "Lightweight Key Management in Wireless Sensor Networks by Leveraging Initial Trust. Particular Report," SRI-SDL04-02, SRI Int'l 2004.

21. S. Capkun, , L. Buttyán, J. P. Hubaux, "Self-Organized Public Key Management for Mobile Ad Hoc Networks," IEEE Trans. Adaptable Computing, Vol. 2, No. 1, 52-64, 2003.

22. S. Chang, , Y. Qi, H. Zhu, J. Zhao, X. Shen, "Impression: distinguishing Sybil strikes in urban vehicular frameworks," IEEE Transactions on Parallel and Distributed Systems, Vol. 23, No. 6, 1103-1114, 2012.

23. S. Park, B. Aslam, D. Turgut, C. C. Zou, "Hindrance against Sybil strike in the fundamental association period of vehicular extraordinarily delegated framework reliant on roadside unit support," Security and Communication Networks, Vol. 6, No. 4, 523-538, 2013.

24. T. Zhou, , R. R. Choudhury, P. Ning, K. Chakrabarty, "P2DAP-Sybil Attacks Detection in Vehicular Ad Hoc Networks," Selected Areas in Communications, IEEE Journal, Vol. 29, No. 3, 582-594, 2011

25. M. A. Razzaque, A. Salehi, S. M. Cheraghi, "Security and Privacy in Vehicular Ad-Hoc Networks: Survey and the Road Ahead," Springer Berlin Heidelberg, In Wireless Networks and Securit, 107-132, 2013.

26. A. Studer, E. Shi, F. Bai, and A. Perrig, "Appending together powerful confirmation, denial, and insurance in vanets," in Conference on SEnsor, Mesh and Ad Hoc COmmunications and Networks (SECON), 2009.

27. J. Newsome, E. Shi, D. Tune, and A. Perrig, (2004)"The Sybil strike in sensor frameworks: Analysis and protections", In Proceedings of International Symposium on Information Processing in Sensor Networks, pp. 259268.

28. F. Anjam, P. Mouchtaris, (2007)"Security For Wireless Ad Hoc Networks", Proc. Intersience Publishing, IEEE.

29. Demirbas, M. likewise, Song, Y., (2006)"An RSSI-based arrangement for sybil strike area in remote sensor frameworks," Proc. WOWMOM

30. B. Xiao, B. Yu, and C. Gao, (2006)" Detection and constraint of Sybil center points in VANETs". In Proceedings of the Workshop on Dependability Issues inWireless Ad Hoc Networks and Sensor Networks (DIWANS '06), Los Angeles, CA, USA, pp. 1-8.
31. Erdogan, S. additionally, Hussain, S., (2007) "Using got signal quality assortment for essentialness powerful data dispersing inWireless Sensor Networks", Proc. DEXA workshop, 620-624.

32. S. Goel, M. Robson, M. Polte, and E. G. Sirer Herbivore, (2003) "A Scalable and Efficient Protocol for Anonymous Communication". Particular Report 20031890, Cornell University, February.

\section{AUTHORS PROFILE}

B.V.S.P.Pavan Kumar , Professor Malla Reddy Engineering College For Women Hyderabad.

Email ID: bvsppkumar@gmail.com

Dr. S.S.V.N.Sharma, Professor, Vagadevi Engineering College, Warangal. Email ID: ssvn.sarma1@gmail.com 